\title{
Research on Intelligent Patrol System of Electric Power Communication Based on Dispatching Intensification
}

\author{
Zhiyuan An ${ }^{1, a}$,Fangfang Dang ${ }^{1, b}$, Lijie Wu ${ }^{1, c}$, Yi Yang ${ }^{1, d}$ \\ ${ }^{1}$ Information \& Telecommunication Co. of State Grid Henan Electric Power Company, \\ Zhengzhou 450052 China

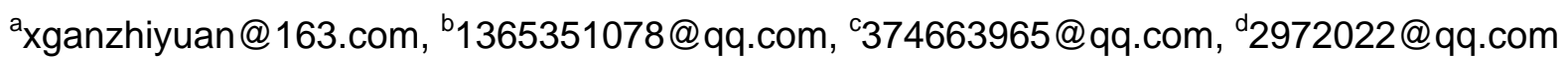

Keywords: dispatching intensification; Intelligent patrol system; information interaction; patrol platform; safety protection;inspection program

\begin{abstract}
The paper presents a design of Electric Power Communication Patrol System based on Dispatching Intensification. The system overall architecture, system security architecture, and system function architecture are elaborated in detail. The system overall architecture mainly explains the perception layer, the network layer, and the application layer. Each layer has a security protection. Business functions include on-site maintenance management, on-site troubleshooting, site inspection management, real-time monitoring, mobile electronic work order management and inspection plan management are all introduced. This system helps maintenance stuffs interact with Communication Dispatching Center with all kinds of collected data, seamlessly connect the operation site and background application. Therefore the security and accuracy of electric power patrol is improved, the efficiency of information-communication dispatching intensification, the problems of human resource shortage and low informatics level is remitted. The system is quite instructive to Electric Power Communication Patrol.
\end{abstract}

\section{Introduction}

Electric power communication patrol aims to detect the defects of equipment and system by analyzing the equipment operating conditions and the surrounding environment on the field. It is a basic work that effectively protects the security of Electric Power System ${ }^{[1]}$.

Nowadays, relative workers still patrol by labor. However, the contradiction between the rapid growing quantity of electric power communication equipment and the shortage of specialized man is increasingly obvious, the difficulty of patrol is increasingly heavily. As the development of Internet technique and Intelligent Terminal Device, the Intellig net Patrol System is capable of remote data collection, transportation, and operation; along with recording the information and of equipment operating conditions and safety on the field, fully functional of patrol and information inquiry etc ${ }^{[2]}$. Therefore, with the trend of Information and Communication Dispatching Intensification, it is undeniably necessary to build an Intelligent Patrol System of Electric Power Communication.

\section{Status Analysis of Telecommunication Patrol}

With the development of Information and Communication Dispatching Intensification, Telecommunication Network is the secondary network beneath the Electric Power Network. The operation and maintenance management therefore become more and more important. Patrol of the field is the most efficient way to locate and process the bugs. Nevertheless, the status of Telecommunication Patrol still has shortages as follows,

\section{Extremely shortage of Human Resources}

The organization of operation and maintenance of existing Electric Power industry is divided by different domain.In recent years, the numbers of devices and communication network resources increase rapidly, while the growth of the number of communications professionals is relatively slow, resulting in a serious shortage of human resources ${ }^{[3]}$. 


\section{The low level of front-line maintenance informationize}

For the characteristics of multi-nodes and wide-distribution in communication network, inspection work always stay with the power transmission line, for quite a long time people checking with the traditional manual filling and artificial statistics. It is far from efficiency and accuracy. It is not conducive to data management and statistical analysis. At the same time, operations staffs only have one simple complete troubleshooting way, telecommunication resources are hard to share. The tradition way goes against bug-treatmenttimely and effectively ${ }^{[4]}$.

\section{Field ops hard to appraisal}

As communications covering the region of thousands of kilometers of transmission lines, hundreds of the substation, numbers of communications facilities, such as maintenance and optimization is difficult to guarantee the quality. Whether the maintenance work is completed as required still lacks of effective monitoring methods at present.

\section{Maintenance working ticket lacks of first-line feedback}

In maintenance work, the dispatching superior and field operations are disjoints, the existing electronic operations process can't form a complete data stream or workflow at the scene. Information stream is not flowing. It is not conducive to business processes, work order flow control, therefore the overall scheduling intensive level is not high.

\section{The operation standardization implement is non-effectively}

Field operation standardized is animportant measures of communications operations work standardization, sequencingand standardization. Each operators has their own working habit and experience, which is non-standard easily lead to unsafe factors, potential safety hazard, and even cause an accident.

Aiming at these problems, such an intelligent inspection system based on the scheduling of the intensification of electric power communication is urge to be built. The system uses information technology and wireless technology to realize intelligent mobile terminal on the scene information collection, field patrol operations for electric power communication terminal management information seamless connection, makes the operation field and background application process seamless integration, realizes the information interaction, improves the timeliness, accuracy of field work data, this system effectively supports the field patrol, maintenance, and troubleshooting, etc.

\section{The System Architecture}

\section{The system overall architecture}

Intelligent Patrol System of Electric Power Communication can be divided asthree-layer-structure, which includes perception layer, network layer and application layer. The perception layer provides information collection; the network layer aims to achieve long-distance transmission of information; the application layer is able to realize the information interaction between patrol system and SGTMS. The structure is shown as figure 1.

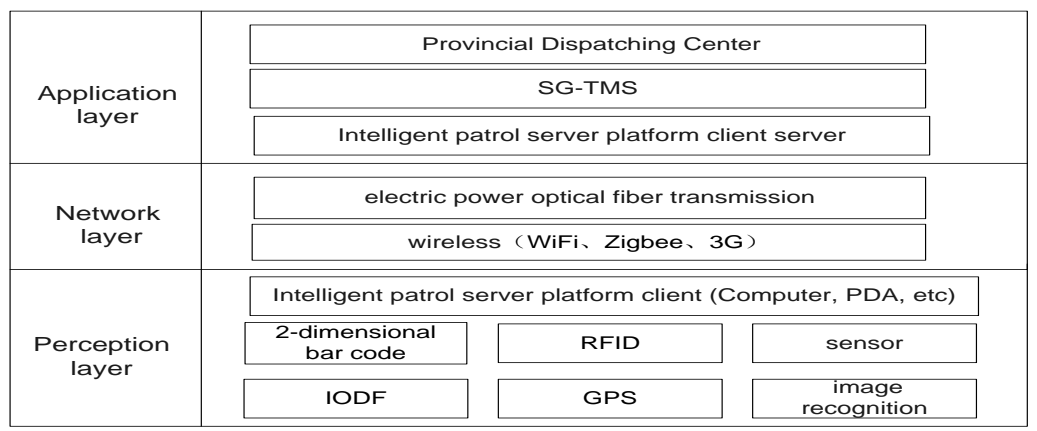

Fig.1: The structure of the system

(1) The perception layer

Intelligent patrol server platform covers both client and server. The platform generally adopts client/server architecture. The client is portable computer, PDA, etc., integrates all kinds of 
information acquisition function, processes variety kinds of information in different formats and protocolsto obtain the original data, andconverses the data into unified message format that suitable for transmission by increasing information head and tail and data encryption protocol. The client using bar code identification technology, RFID(radio frequency identification) technology, sensor technology, iODF(intelligent optical fiber distribution frame)technology, GPS technology and image recognition technology to provide upper layer with the underlying data ${ }^{[5] \sim[6]}$, includes the name of stations, name of the machine rooms, equipment types, fiber length, the fiber topology path, and the equipment statusetc., providing all-weather and all-around information support for production.

(2) The network layer

The Network layer realizes all kinds of measurement, feeling, dispatching, information, and control such as collecting information transmission between the perception layer and application layer. The inspection platform client and server WebServiceare based on HTTP protocol. According to different environment, power communications intelligent inspection platform client will transmit perceive layer informationto inspection platform server in different ways. Due to the long transmission distance, it is adjustable to use a combination of wireless and wired, such as Wi-Fi, ZigBee, 3G, and electric power optical fiber transmission mode. While platform is security accessed, remote data transmission and receiving is implemented.

(3) the application layer

Intelligent patrol server platform achieves data synchronization service, user authentication service, mobile terminal management, integrated management system and the interaction between patrol system and SG-TMS, while providing a standard service interface to the client of intelligent patrol platform. The system server and application layer network servers, and SG-TMS are deploymented in Provincial Dispatching; using TCP protocol to realizes the interaction.

\section{System security architecture}

Power communication intelligent inspection platform security architecture can be divided into the perception layersecurity protection, network layer security protection and application layer security protection, as shown in figure 2. Mainly focus on security of mobile terminal applications, storage, security, and the loss of the client device security, as well as the reliable server access, illegal terminal intrusion security problems ${ }^{[7]}$.

(1) The perception layer security protection

Perception layer security protection ,AKA security terminals layer, the data of electric power intelligent patrol server platform client is stored in encryption card. Thus, the sensitive data is encrypted by running the safety management of the client, ensure the safety of the raw data on your mobile phone, therefore information leakage or being tampered with can be avoid.

(2) The network layer security protection

The network layer security protection includes safe passage layer and security access platform layer. When the intelligent inspection platform client accesses to the application server, the client provides security access to reliable VPN network serverthrough the security platform rather than directly visit. After authentication though trusted security access, bidirectional encryption channel is established, the safety of data transmission is ensured. Through the security authentication, digital certificate, and other means the mobile device access is controlled. The platform also capable of security access gateway, data filtering exchange system, identity certification server and access platform centralized monitoring management system to provide security protection.

(3) The application layer security protection

Application layer security, also known as patrol platform server layer, by checking the user name, user password and equipment unique identification authentication mechanism, controls user access, thus ensure the security of the application layer. The security structure is shown as figure 2. 


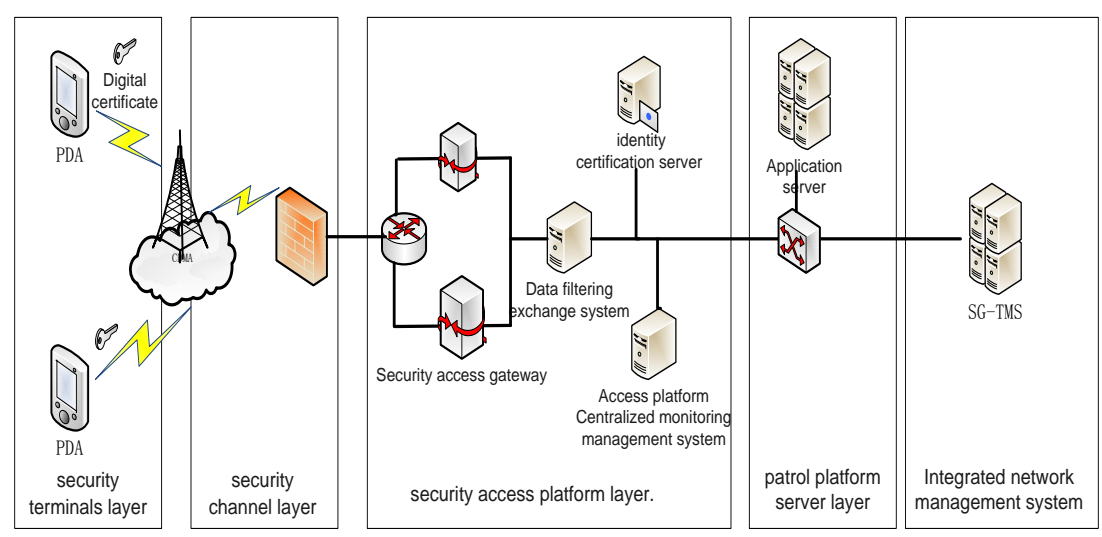

Fig.2: The security structure of the system

\section{The system function architecture}

The function scope of Intelligent Patrol System of Electric Power Communication is divided into: basic function, business function and safety protection function. the basic function is implemented by the system platform. Business functions include on-site maintenance management, on-site troubleshooting, site inspection management, real-time monitoring, mobile electronic work order management and inspection plan management. Safety protection is mainly composed by security access platform to realize, the functional architecture are shown in figure 3 below:

\begin{tabular}{|c|c|c|c|}
\hline $\begin{array}{l}\text { basic } \\
\text { function }\end{array}$ & \multicolumn{3}{|c|}{ the system platform } \\
\hline \multirow{2}{*}{$\begin{array}{l}\text { business } \\
\text { function }\end{array}$} & $\begin{array}{c}\text { on-site maintenance } \\
\text { management }\end{array}$ & on-site troubleshooting & $\begin{array}{r}\text { site inspection } \\
\text { management }\end{array}$ \\
\hline & $\begin{array}{c}\text { Real-time monitoring } \\
\text { management }\end{array}$ & $\begin{array}{c}\text { mobile electronic work } \\
\text { order management }\end{array}$ & $\begin{array}{r}\text { inspection plan } \\
\text { management }\end{array}$ \\
\hline $\begin{array}{c}\text { safety } \\
\text { protection }\end{array}$ & & ecurity access platform & \\
\hline
\end{tabular}

Fig.3: The functionalarchitecture of the system

(1) On-site maintenance management

The on-site patrol stuff acquire information of the station, computer room, equipment, wiring, and wiring information through the perception layer, in order to realize the fast query of maintenance information. While SG-TMS provide intelligent repair work content, maintenance process steps, safety measures, contingency plans and implement maintenance data and automatic input functionfor on-site staff to.

(2) on-site troubleshooting

Operations staff can quickly remote query site, computer room, equipment, lines information, etc, then transfer photographs, videos and other fault information on the real situation in a timely manner to the provincial information communication control center for the remote expert diagnosis. Therefore we have a formation of longitudinal penetration mechanism, providing technical support for on-site troubleshooting and repair, improve the accuracy of the fault handling, finallyprovide technical support for the realization of the electric power communication scheduling management system.

(3) On-site patrol management

Provincial communication control center can deliver the on-site inspection plans to remote handset, on-site patrol stuff execute that plan and send information back to SG-TMS record as informationlized logbooks. At the same time, the provincial communication control center superviseon-site work though GPS, RFID, bio-dimensionpositioning technology, video and other technology, including patrol work is established in accordance with the tour plan and patrol routes, whether or not on-site stuff is in place and working condition. 
(4) real-time monitoring management

Provincial control center is access to accurate and timely monitoring data, alarm push, alarm processing and other functions from the integrated network management system, SG-TMS and service of the patrol system platform.

(5) Mobile electronic work order management

The system is integrated with SG-TMS, providing mobile electronic work order checking, examination and approval, and other functions such as associating with voicephone and SMS, implemented the task distribution function. Once the inspection stuff noticedan equipment trouble, repair process can be active immediately through the inspection platform client.

(6) inspection plan management

Patrol work plan management is aimed at all kinds of operational planning work of electronic management, communication management stuff can customize reports according to the inspection plan, and carry out with the help of inspection system. The inspectionoutlines management, annual inspection plans, monthly plans etc. are also customized though the system. According to annual inspection plan outline, subordinateset up the annual inspection and monthly inspection plans, respectively, report to the superior unit for examination, approval, and implement.

\section{System Application}

The application of Intelligent Patrol System of Electric Power Communication can be divided asthree part which includes perception layer deployment, the network layer deployment and the application layerdeployment, show as figure 4.

(1) perception layer deployment

Perception layer deployment is to complete the installation and debugging of client, such as stations, computer rooms, equipment, intelligent inspection platform hardware. Pastedifferent RFIDand bar code according to the different equipment. Complete deployment of sensors, GPS and iODF. Then test the information acquisition function of the platform client, guaranteethe data accuracy and reliability.

(2) the network layer deployment

Network layer deployed establish the network structures, and security access configuration, achieve conversion between cable and wireless transmission mode by protocol converter. Through the variety of authentication server at province side to ensure data security, complete. Finally, test the interconnected channels and platform security access.

(3) the application layerdeployment

Establish the server of intelligent patrol system and every function module; realize the information interaction between SG-TMS and the application server. At last the provincial information communication control center collect the information of subordinatecompany for testing, ensure the realization of the system function of each business.

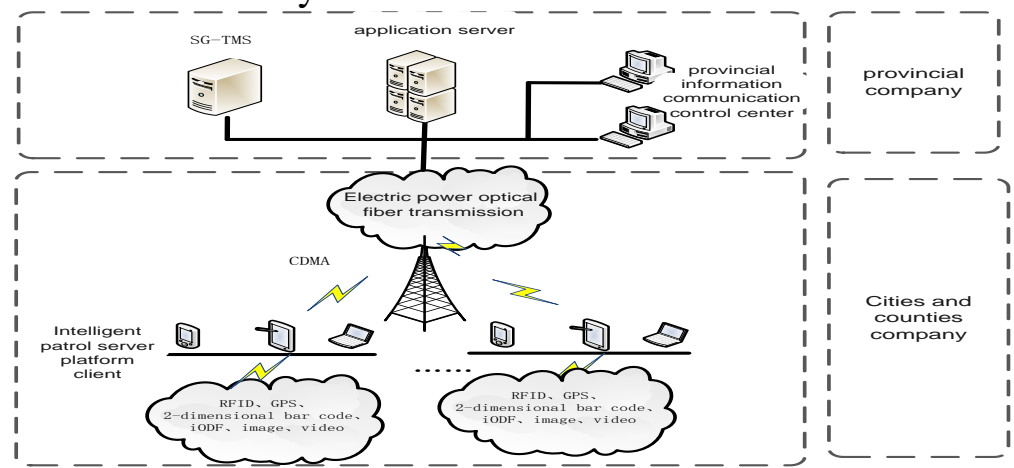

Fig.4: The application of the system 


\section{Conclusions}

Intelligent Patrol System of Electric Power Communication based on Dispatching Intensification is centralized deployment in provincial control center, effectively save first-line operations staff, and integrate provincial and cities'dispatching resources, form a longitudinal penetration mechanism. By developing Intelligent Patrol System of Electric Power Communication , the traditional way of paper records is changed ,operating efficiency is improved, reduce the missing rate, ensure the patrol tour in place and its specification. Finally realize the closed-loop management of data of field work, improve information communication intensive scheduling management, solve the shortage of human resources, and operational work order process lack of field part, ideally meets the demand of the development of intelligent grid power communication networks, resulting in good economic and social benefits.

\section{References}

[1] Zhang Ting, Design of Power Line Inspection and Field Failure Diagnosis System based on GNSS . Electronic Design,2013（22）,pp:142-143,2013.

[2] Zhou Hong-Yu,Design and Implementation of Intelligent Inspection System Communication Module based on B/S,Beijing:Beijing University of Posts and Telecommunications,2011.

[3] Sun Jie,Discussion of the variable standard mode of electricity operation based on the integration, China Electric Power Education 2012(18),pp:102-103,2012.

[4]Chen Xuan,The Information Technology of Power Communication NetworkOperation, Technology Economy ,2010(9),pp:24-25,2010.

[5]Lin Wen-huan,Design and Implementation of Iintelligent Information Systems based on Mobile Terminals ,Xi’an: Xi`an Jiaotong University,2011.

[6]Li Gui-mao,Research and implementation of intelligent inspection system based on GPS and GIS,Changsha: Central South University, 2011.

[7]Zhang Zhou-feng, security analysis and architecture of Intelligent inspection system based on RFID,Zhengzhou: Zhengzhou University, 2011. 